Revisión de tema

\title{
Candidemia en Colombia
}

\author{
Jorge Alberto Cortés ${ }^{1,2}$, José Franklin Ruiz³ ${ }^{3}$ Lizeth Natalia Melgarejo-Moreno², \\ Elkin V. Lemos ${ }^{3}$ \\ ${ }^{1}$ Departamento de Medicina Interna, Facultad de Medicina, Universidad Nacional de Colombia, \\ Bogotá, D.C., Colombia \\ ${ }^{2}$ Grupo de Investigación en Enfermedades Infecciosas, Facultad de Medicina, Universidad \\ Nacional de Colombia, Bogotá, D.C., Colombia \\ ${ }^{3}$ Departamento Médico, Pfizer, Bogotá, D.C., Colombia
}

En Colombia, especialmente en las unidades de cuidados intensivos, la candidemia es una causa frecuente de infección del torrente sanguíneo y representa el $88 \%$ de las infecciones fúngicas en pacientes hospitalizados, con una mortalidad entre 36 y $78 \%$. Su incidencia en Colombia es mayor a la reportada en los países desarrollados e, incluso, en otros países de Latinoamérica. Para su manejo deben considerarse los factores de riesgo del paciente, luego valorar las características clínicas y, finalmente, hacer los estudios microbiológicos y, si es necesario, pruebas moleculares.

En general, las guías estadounidenses, latinoamericanas y europeas recomiendan las equinocandinas como el tratamiento de primera línea de la candidemia y difieren en el uso de fluconazol dependiendo de la 'evidencia', la gravedad de la enfermedad, la exposición previa a los azoles y la prevalencia de Candida no albicans. Dada su gran incidencia en nuestro país, asociada con una elevada mortalidad, esta infección debe buscarse sistemáticamente en pacientes con factores de riesgo, con el fin de iniciar oportunamente el tratamiento antifúngico.

Palabras clave: candidiasis; candidemia; unidad de cuidados intensivos; micosis; infecciones fúngicas invasivas; candidiasis invasiva; Colombia.

\section{Candidemia in Colombia}

In Colombia, especially in intensive care units, candidemia is a frequent cause of infection, accounting for $88 \%$ of fungal infections in hospitalized patients, with mortality ranging from $36 \%$ to $78 \%$. Its incidence in Colombia is higher than that reported in developed countries and even higher than in other Latin American countries. First, the patient's risk factors should be considered, and then clinical characteristics should be assessed. Finally, microbiological studies are recommended and if the evidence supports its use, molecular testing. In general, American, Latin American, and European guides place the echinocandins as the first-line treatment for candidemia and differ in the use of fluconazole based on evidence, disease severity, previous exposure to azoles, and prevalence of Candida non-albicans. Taking into account the high incidence of this disease in our setting, it should be looked for in patients with risk factors to start a prompt empirical anti-fungal treatment.

Recibido: 31/04/2018

Aceptado: 07/06/2019

Publicado: 19/07/2019

Citación:

Cortés JA, Ruiz JF, Melgarejo-Moreno LN, Lemos EV. Candidemia en Colombia. Biomédica. 2020;40:195-207.

https://doi.org/10.7705/biomedica.4400

\section{Correspondencia:}

Jorge Alberto Cortés, Departamento de Medicina Int erna, Facultad de Medicina, Universidad Nacional de Colombia, Carrera 30 No 45-03, oficina 510, Cuidad Universitaria, Bogotá, D.C., Colombia

Teléfono: (571) 3165000 , extensión 15011

jacortes!@unal.edu.co

\section{Contribución de los autores:}

Jorge Alberto Cortés y José Franklin Ruiz: diseño y estructura inicial de la revisión

Todos los autores participaron en la escritura general y la revisión final.

Financiación:

El presente estudio no tuvo financiación directa.

Conflicto de intereses:

E. V. Lemos y J. F. Ruiz son empleados de Pfizer; J. A. Cortés tiene apoyo no financiero de Pfizer y Merck Sharp and Dohme, y L. N. Melgarejo-Moreno informa que no tiene conflicto de intereses.
Keywords: Candidiasis; candidemia; intensive care units; mycoses; invasive fungal infections; candidiasis, invasive; Colombia.

El aumento de la población de adultos mayores, pacientes oncológicos, con trasplante e inmunocomprometidos, implica un crecimiento significativo de la población en riesgo para el desarrollo de complicaciones infecciosas, entre ellas la candidemia, cuya incidencia aumenta de manera notoria en la población críticamente enferma. Hasta el $85 \%$ de las infecciones fúngicas en las unidades de cuidados intensivos corresponde a infecciones por Candida spp. (1) y su mortalidad es cercana al $40 \%$ (2). Por otro lado, está la resistencia a los antifúngicos disponibles para el tratamiento de la candidemia: en Bogotá se ha establecido la resistencia al fluconazol hasta en $30 \%$ de los aislamientos (1) y, en Medellín, en el $22 \%$ de los casos (3).

El objetivo de la presente revisión fue compilar la información disponible en los últimos 10 años sobre la candidemia en Colombia.

\section{Candidemia}

La candidiasis, es decir, la infección producida por las levaduras del género Candida, comprende un espectro clínico que abarca infecciones superficiales y diseminadas que afectan cualquier órgano o sistema. Las 
especies de Candida son parte de la flora normal del cuerpo humano, pero solo un pequeño porcentaje de las especies identificadas causan enfermedad. Candida albicans es responsable de la mitad de los casos (4), aunque su frecuencia parece estar disminuyendo en los últimos años $(5,6)$.

La candidiasis invasiva ocurre cuando las levaduras del género Candida alcanzan el torrente sanguíneo, desde donde pueden diseminarse a cualquier tejido de la anatomía humana. La candidiasis invasiva incluye un amplio espectro: candidemia con endoftalmitis o sin ella, infecciones hematógenas diseminadas, compromiso de un órgano (infecciones abdominales, peritonitis, meningitis y endocarditis) y candidiasis hepatoesplénica, principalmente en pacientes con enfermedad hematológica. La fuente de infección por Candida spp. puede ser endógena, de la flora gastrointestinal o de la colonización mucocutánea, o exógena, de las manos de los trabajadores de la salud o de objetos o infusiones contaminadas (7). La candidemia es la forma clínica de la candidiasis invasiva que ocurre más frecuentemente. La frecuencia de las diferentes especies de Candida en esta infección varía geográficamente y en el tiempo, como se explicará posteriormente.

\section{Factores de riesgo}

El principal factor de riesgo es la gravedad de la condición de base del paciente, la cual usualmente se valora con la puntuación de la Acute Physiology and Chronic Health Evaluation II (APACHE II), cuyo uso ha sido validado en las unidades de cuidados intensivos, aunque en muchos estudios se ha usado para todos los pacientes.

La gravedad de la enfermedad implica factores adicionales de riesgo, como el uso de antibióticos de amplio espectro, las cirugías mayores, la nutrición parenteral total y el uso de dispositivos invasivos como sondas y catéteres (8). Otros factores de riesgo descritos son la duración de la estancia hospitalaria, la edad avanzada, la presencia de múltiples comorbilidades, la diabetes mellitus, los trasplantes, las enfermedades neoplásicas, la cirugía gastrointestinal, los implantes protésicos, los tratamientos inmunosupresores, la pancreatitis, la desnutrición, las quemaduras graves, el trauma, la colonización previa por Candida spp., la falla renal aguda -principalmente en pacientes con diálisis-, el compromiso del sistema inmunológico, el bajo peso al nacer, la neutropenia, la hospitalización en unidades de cuidados intensivos, la disfunción orgánica que requiere procedimientos invasivos como la asistencia respiratoria mecánica, los medicamentos vasoactivos, la diálisis, la circulación extracorpórea, las transfusiones y la traqueotomía, entre otros (cuadro 1) $(2,7,9,10)$. Un estudio realizado en tres instituciones de diferentes partes de Colombia, validó la frecuencia e importancia de los factores de riesgo mencionados (11).

Uno de los factores de riesgo más relevantes para las candidiasis invasivas es el uso de antimicrobianos. Los antibióticos tienen varios efectos que favorecen la multiplicación de las levaduras, entre ellos, la eliminación de la microbiota nativa que establece un control ecológico sobre las especies de Candida presentes en el tubo digestivo y la limitación de la diversidad de especies que se encuentran en un momento dado. Aunque algunos antibióticos se han asociado más que otros con infecciones por Candida spp. (11), cualquiera de amplio espectro puede tener este efecto. 
Cuadro 1. Factores de riesgo para candidemia en pacientes hospitalizados

\begin{tabular}{ll}
\hline Factor & \multicolumn{1}{c}{ Condicionantes } \\
\hline Tiempo de hospitalización & Exposición a múltiples factores de riesgo \\
Microbiota colonizadora & Tratamiento con antibióticos \\
& Colonización por Candida \\
Inmunosupresión & Problemas de control de infecciones \\
& Quimioterapia \\
& Corticoesteroides \\
& Cáncer \\
Procedimientos médicos invasivos & Neutropenia \\
& Prematuridad \\
& Respiración mecánica asistida \\
& Cirugía \\
& Hemodiálisis \\
& Nutrición parenteral \\
\hline
\end{tabular}

\section{Fisiopatología}

Las levaduras de Candida spp. viven normalmente en el cuerpo sin causar daño. Cuando hay compromiso de la inmunidad o una condición que conlleve el desequilibrio de la flora normal, la cantidad de levaduras aumenta produciendo su colonización excesiva y el paso al torrente sanguíneo mediante translocación (9). Los pacientes con cirugía abdominal y nutrición parenteral tienen mayor riesgo de infecciones de origen endógeno debido, en ambos casos, a la alteración de la fisiología gastrointestinal y la consecuente pérdida del epitelio o de sus uniones, lo cual favorece el paso desde la luz intestinal hasta los vasos sanguíneos. Lo mismo sucede en los pacientes con neutropenia posterior a la quimioterapia, la cual produce lesiones importantes del epitelio que se manifiestan como diarrea y mucositis, y se refleja en otros epitelios como el cuero cabelludo o las células hematopoyéticas (9).

Por otro lado, las fuentes de infección exógena incluyen la contaminación de las manos del personal de atención, de las soluciones parenterales y oftálmicas, y de dispositivos como catéteres, válvulas cardíacas y respiradores, entre otros (12).

En un estudio en unidades neonatales de cuidados intensivos de Bogotá, se evidenció la frecuencia elevada de infección ocasionada por el mismo tipo de especies de Candida identificadas como colonizadoras en los pacientes (13), así como el de un brote relacionado con problemas de control de infecciones en estas unidades, en las que se comprobó la colonización de catéteres centrales y la posterior infección debido a deficientes medidas de limpieza y desinfección de los puertos endovenosos (14). Además, se ha estimado que, en condiciones de cuidados intensivos, más del $30 \%$ de los casos observados estarían relacionados con brotes (15).

Entre los factores de virulencia que determinan la relación de Candida spp. con el huésped, se ha demostrado su capacidad de adherirse a los tejidos y a las superficies de catéteres o prótesis, lo que les permite colonizar y formar una biopelícula que limita la acción de los antimicrobianos. La biopelícula frena el metabolismo y el crecimiento del hongo, lo cual disminuye los sitios de acción de la mayoría de los antifúngicos (9). Asimismo, se ha establecido que las biopelículas ayudan al incremento de los genes de resistencia de las levaduras (16). Otros factores determinantes de la diseminación e invasión de los tejidos son la producción de enzimas 
(proteasas, fosfolipasas y lipasas) y el cambio morfológico de Candida spp. de blastoconidia o seudohifa a hifa (12).

\section{Epidemiología en Colombia}

La frecuencia de la candidemia varía según los servicios de hospitalización y los factores de riesgo de los pacientes. Además, se han observado cambios en la epidemiología de las especies de Candida en cuanto a la incidencia y la resistencia a los antifúngicos por área geográfica (12). En un estudio epidemiológico de siete países latinoamericanos que incluía pacientes colombianos, se reportó una incidencia general de candidemia de 1,18 casos por cada 1.000 admisiones en el conjunto de hospitales incluidos en el estudio.

En Colombia se reportó la incidencia más alta: 1,98 casos y, en Chile, la más baja: 0,33 casos por 1.000 admisiones (5). Estos datos contrastan con los de tasas más bajas de incidencia de candidemia reportadas en los Estados Unidos, con 0,96 casos, y en Europa, con 0,38 casos por 1.000 admisiones (2).

En un estudio de siete hospitales de Colombia entre el 2004 y el 2008, se reportó que la frecuencia de candidiasis invasiva era superior a la establecida en países desarrollados. La incidencia fue de 2,3 casos por 1.000 días de estancia en una unidad de cuidados intensivos, con una incidencia global observada de 1,4\% por egresos de la unidad (17). Se observó una tendencia al aumento de la incidencia y la prevalencia de candidemia, principalmente por especies de Candida no albicans, las cuales correspondieron a $44 \%$ de los aislamientos.

Esta dinámica epidemiológica tiene implicaciones importantes en el tratamiento, dada la menor sensibilidad de estas especies al fluconazol (17). Las razones de los cambios de la incidencia, así como de los cambios epidemiológicos observados en Colombia, no son claras y ameritan estudios apropiados para entender su dinámica. Según un estudio de la Corporación para Investigaciones Biológicas (CIB) de Medellín, la candidiasis invasiva representaba el $75 \%$ de las infecciones por hongos en pacientes hospitalizados, con una mortalidad asociada del $78 \%$ (12).

La disparidad de las cifras en los estudios citados puede explicarse por diferencias en las características demográficas, en la práctica clínica y en las comorbilidades. Sin embargo, la verdadera incidencia de candidiasis invasiva puede ser mayor debido al alto porcentaje de cultivos falsos negativos (30 a $50 \%$ ) y a la dificultad del diagnóstico de candidiasis invasiva sin candidemia, como se verá más adelante (7).

En los últimos años, se ha observado a nivel mundial un incremento de la frecuencia de una especie emergente: $C$. auris. Este microorganismo se ha detectado en Asia, Europa, Estados Unidos y Suramérica (18). En Colombia, se han documentado brotes en la Costa Atlántica y se han observado casos en ciudades andinas (19), así como brotes en algunos hospitales (20). En el primer brote reportado, hubo una gran mortalidad en los 17 pacientes con infección por esta levadura (21). Los factores de riesgo establecidos no parecen diferir mucho de los ya reportados, aunque aún no es claro por qué este microorganismo, previamente desconocido, se ha diseminado a nivel mundial.

La frecuencia de las diferentes especies de Candida en Colombia se presenta en el cuadro $2(1,3-6,11,22)$. Se observa que los datos tomados en su conjunto reflejan una disminución de la frecuencia relativa de $C$. albicans, 
Cuadro 2. Distribución de especies de Candida en estudios colombianos de pacientes con infección del torrente sanguíneo

\begin{tabular}{|c|c|c|c|c|c|c|c|}
\hline $\begin{array}{l}\text { Estudio y años de } \\
\text { recolección }\end{array}$ & $\begin{array}{c}\text { Zuluaga-Rodríguez, } \\
\text { et al. (3) 2001-2007 } \\
\text { n (\%) }\end{array}$ & $\begin{array}{c}\text { Cortés, et al. } \\
\text { (4) 2001-2007 } \\
\text { n (\%) }\end{array}$ & $\begin{array}{c}\text { Cortés, et al. } \\
\text { (1) 2008-2009 } \\
n(\%) \\
\end{array}$ & $\begin{array}{c}\text { Nucci, et al. } \\
\text { (5) 2008-2010 } \\
n(\%) \\
\end{array}$ & $\begin{array}{c}\text { Ortiz, et al. } \\
\text { (11) 2008-2012 } \\
n(\%)\end{array}$ & $\begin{array}{c}\text { Berrío, et al, } \\
\text { (6) } 2010-2011 \\
\mathrm{n}(\%) \\
\end{array}$ & $\begin{array}{c}\text { Motoa, et al. } \\
\text { (22) 2010-2013 } \\
\mathrm{n}(\%) \\
\end{array}$ \\
\hline Candida albicans & $147(43,6)$ & $214(56)$ & $87(66,4)$ & $253(37,6)$ & $42(52)$ & $60(40,8)$ & $21(41,2)$ \\
\hline Candida tropicalis & $79 \quad(23,4)$ & $66(17,3)$ & $14(10,6)$ & $118(17,6)$ & $14(17)$ & $25(17)$ & $9(17,6)$ \\
\hline Candida parapsilosis & $47 \quad(13,9)$ & $61(16)$ & $19(14,5)$ & $178(26,5)$ & $14(17)$ & $34(23,1)$ & $7(13,7)$ \\
\hline Candida glabrata & $32 \quad(9,5)$ & $10(2,6)$ & $2(1,5)$ & $42(6,3)$ & $5(6)$ & $10(6,8)$ & $4(7,9)$ \\
\hline Candida krusei & $(3,3)$ & $3 \quad(0,8)$ & 0 & $18(2,7)$ & $2(3)$ & $3(2,04)$ & 0 \\
\hline
\end{tabular}

con incremento de otras especies, especialmente C. parapsilopsis. También, se ha observado un incremento relativo de la frecuencia de $C$. glabrata. No es claro el significado de estos cambios, aunque algunas especies, como $C$. parapsilosis, parecen tener mayor facilidad para colonizar a los trabajadores de la salud y formar biopelículas, lo cual favorecería su transmisión cruzada y podría explicar parcialmente los cambios observados $(13,14,23)$.

La candidemia puede presentarse sobre todo en dos situaciones: usualmente, en unidades de cuidados intensivos en pacientes sin neutropenia críticamente enfermos y con factores predisponentes y, la segunda, en pacientes con enfermedad oncológica y con neutropenia secundaria a la quimioterapia, generalmente por tumores hematológicos (4). En los estudios realizados en los hospitales de Colombia, entre 37 y $45 \%$ de los pacientes estaban hospitalizados en unidades de cuidados intensivos y, en una cuarta parte de ellos, el cáncer era el factor de riesgo predominante (2).

En otro estudio en el país, se encontró que la candidemia era la quinta causa de infecciones del torrente sanguíneo y la infección fúngica invasiva más común, y que era responsable de cerca del $75 \%$ de todas las micosis invasivas en los hospitales $(12,24)$, con el consecuente aumento de los costos, la morbilidad y la mortalidad, especialmente en las unidades de cuidados intensivos (25). Se estima que más del $10 \%$ de las infecciones asociadas con la atención en salud son causadas por Candida spp. y que cerca de la mitad de estas ocurren en dichas unidades. La incidencia de la candidiasis invasiva en estas unidades es diez veces mayor que en los servicios quirúrgicos o de hospitalización (25). El costo estimado de cada episodio de candidiasis invasiva en adultos hospitalizados es de aproximadamente USD $\$ 40.000$ (26) y, en Colombia, los costos totales de atención de pacientes con candidemia equivalieron a COP\$ 6.437’210.186 en el 2013 (27).

\section{Diagnóstico}

La presentación clínica se caracteriza por síntomas inespecíficos; las manifestaciones más comunes son fiebre, escalofríos y otros signos de reacción inflamatoria que no mejoran con la terapia antibiótica. Si la infección se extiende a otros órganos, por ejemplo, riñones, hígado, huesos, músculos, articulaciones, bazo u ojos, se desarrollan síntomas determinados según el sitio de la infección. Además, el paciente puede presentar falla orgánica.

En un estudio en el país, se estableció que el $83 \%$ de los pacientes presentaba inflamación en el momento de la detección de la candidemia, $25 \%$ tenía disfunción de tres o más órganos y $34 \%$ se encontraba en choque hemodinámico (1). Dado el impacto que tiene en el pronóstico de la salud visual, la selección del antifúngico y la duración del tratamiento, es muy importante detectar la infección ocular en los casos de candidemia. 
En los pacientes críticamente enfermos, el diagnóstico de candidemia no es fácil, dado que los signos y los síntomas varían drásticamente y que los procedimientos diagnósticos existentes tienen serias limitaciones (7).

El diagnóstico se basa en la comprobación mediante métodos microbiológicos, examen directo, histopatología, cultivo de sitios estériles (que requieren procedimientos invasivos) o mediante pruebas serológicas para anticuerpos y antígenos, y moleculares para detectar el ADN de Candida spp. (12). Los hemocultivos continúan siendo la piedra angular del diagnóstico, sin embargo, su sensibilidad es poca (30 a $50 \%$ ) y requieren largos periodos de incubación (28). Los nuevos métodos de cultivo tienen mayor capacidad de detección de Candida spp. (70\%), pero requieren mínimo de 24 a 48 horas para mostrar positividad, por lo que el resultado puede ser tardío (29).

El diagnóstico temprano es necesario, ya que el inicio tardío del tratamiento se asocia con aumento de la mortalidad. Se deben considerar primero los factores de riesgo del paciente, luego valorar las características clínicas y, por último, adelantar los estudios microbiológicos (29). Este proceso implica recurrir al tratamiento empírico basado en los factores de riesgo y los índices de predicción de candidiasis. Los más usados en nuestro medio son el índice de colonización de Pittet y el puntaje de Candida (o índice de León) (30). La utilidad de estos índices está determinada por su valor predictivo negativo, ya que la posibilidad de candidemia es extremadamente baja, con índices negativos en pacientes sin neutropenia (24).

Para un diagnóstico más rápido y oportuno, se han desarrollado marcadores serológicos que detectan componentes de la pared celular del hongo, como los manano-oligosacáridos y el 1,3- $\beta$-D-glucano. Estos marcadores no se utilizan rutinariamente en nuestro medio. En la candidiasis invasiva, los hemocultivos y la reacción en cadena de la polimerasa (PCR) en pruebas combinadas de antígenos y anticuerpos (manano y anticuerpos antimanano) alcanzan una especificidad del 90 al $100 \%$ y una sensibilidad del 30 al $60 \%$ para la detección de Candida spp. (24). Las pruebas moleculares son prometedoras, pero aún son de acceso limitado. Es claro que el diagnóstico requiere de los estudios moleculares de laboratorio, y que las técnicas más novedosas para la identificación de los productos de la PCR aportan mayor sensibilidad y especificidad (31).

\section{Pronóstico}

La candidiasis invasiva conlleva aumento de la mortalidad en el caso del inicio tardío del tratamiento. La ausencia de métodos de gran sensibilidad y especificidad para el diagnóstico rápido, creó la necesidad de determinar los factores de riesgo y recurrir a métodos de evaluación para garantizar el tratamiento oportuno, incluso en ausencia de evidencia microbiológica de infección (7).

La candidemia es un evento terminal en una significativa proporción de pacientes. La tasa de letalidad oscila entre el 40 y el $60 \%$ al mes del episodio y, mayor, en los estudios con seguimiento a un año. La mortalidad a corto plazo sugiere que un episodio de candidemia es un signo ominoso; sin embargo, en ausencia de una enfermedad subyacente grave, la candidemia se considera una condición más benigna (32). En un estudio en hospitales de Bogotá, se informó que la tasa de mortalidad durante la hospitalización alcanzó el 36 \% (1), en tanto que la mortalidad global latinoamericana en un estudio multicéntrico fue de $40 \%$ (2). No se encontraron estudios en nuestro medio que permitieran establecer la mortalidad atribuible a candidemia. 
El principal factor de mejoría del pronóstico es el inicio temprano del tratamiento antifúngico. Si el tratamiento se inicia en el momento de conocer la positividad de los hemocultivos o durante las primeras 12 horas, la mortalidad es de 10 a $15 \%$ y, si su inicio se retrasa 48 horas, la mortalidad asciende a 30 a $35 \%$ (33).

Otro factor asociado con un mejor pronóstico es el retiro temprano del catéter venoso central. Las guías aconsejan que, en los pacientes con candidemia, se retire tan pronto como sea posible $(26,34)$, pues, aunque el acceso venoso no siempre es el origen de la infección, es un reservorio que prolonga la candidemia e incrementa el riesgo de focos metastásicos de infección, por lo que su retiro se asocia con una menor duración y mortalidad por la infección (35).

Otra acción relevante para mejorar el pronóstico es la exploración ocular. Todas las guías recomiendan hacer una exploración del fondo del ojo, dado que se ha reportado la incidencia de endoftalmitis o coriorretinitis por Candida spp. en 5 a $78 \%$ de los pacientes con candidemia (36).

\section{Tratamiento de la candidemia en pacientes sin neutropenia}

La candidemia requiere tratamiento antifúngico durante varias semanas; su tipo y su duración dependen de factores específicos del paciente, como la edad, las comorbilidades, el estado inmunológico y la gravedad de la infección, así como la sensibilidad de la especie identificada (37). Hay diferentes antifúngicos disponibles para el tratamiento de la candidiasis (anfotericina B, flucitosina, azoles), sin embargo, con este grupo de medicamentos se han documentado efectos adversos importantes debidos a su toxicidad o a las interacciones farmacológicas. Más recientemente, se han incorporado las equinocandinas (anidulafungina, caspofungina y micafungina), fungicidas que tienen un amplio espectro de acción con mayores tasas de éxito clínico y son bien tolerados (38).

Es importante resaltar que, entre dichos medicamentos, hay diferencias importantes en el perfil de uso clínico, derivadas de sus características farmacocinéticas particulares (cuadro 3).

En el cuadro 4 se presentan esquemas terapéuticos recomendados para el manejo de la candidiasis invasiva o candidemia en cuatro situaciones, todos ellos formulados en los últimos seis años, siendo la más reciente actualización la de la guía de la Infectious Diseases Society of America (IDSA).

Cuadro 3. Diferencias de los efectos adversos e interacciones farmacológicas de las diferentes equinocandinas

\begin{tabular}{lll}
\hline Caspofungina & Micafungina & Anidulafungina \\
\hline $\begin{array}{l}\text { Interacciones con rifampicina, } \\
\text { fenitoína, carbamazepina, }\end{array}$ & $\begin{array}{l}\text { Ausencia de evidencia de } \\
\text { eficacia y seguridad en falla }\end{array}$ & $\begin{array}{l}\text { No tiene ningún grado de } \\
\text { metabolismo hepático o renal. }\end{array}$ \\
$\begin{array}{l}\text { Requiere ajuste de la dosis en } \\
\text { pacientes con falla hepática } \\
\text { (clasificados como Child-Pough }\end{array}$ & $\begin{array}{l}\text { El reporte de formación de } \\
\text { tumores hepáticos en roedores }\end{array}$ & $\begin{array}{l}\text { No requiere ajuste de dosis } \\
\text { en falla renal o hepática. }\end{array}$ \\
$\begin{array}{l}\text { adecuesconoce la dosis } \\
\text { adeocupación. }\end{array}$ & \\
\hline
\end{tabular}

Adaptado de: Paramythiotou E, et al. Invasive fungal infections in the ICU: how to approach, how to treat. Molecules. 2014;19:1085-119. 
El actual consenso colombiano de práctica clínica para el manejo de la candidemia es una adaptación de las guías internacionales, especialmente, la estadounidense y la europea (39). También, se cuenta con el protocolo de estudio y manejo de pacientes con candidiasis sistémica de la Asociación Colombiana de Infectología (ACIN) (24), el cual se resume en el cuadro 5.

Este protocolo y las guías de manejo privilegian el uso de las equinocandinas (anidulafungina, micafungina y caspofungina), sin que se haya documentado una mayor efectividad de una sobre las otras y con

Cuadro 4. Medicamentos disponibles o recomendados para diferentes situaciones de candidemia

\begin{tabular}{|c|c|c|}
\hline Cuadro clínico & Medicamento de elección & Medicamentos alternativos \\
\hline Candidemia & Caspofungina o anidulafungina & $\begin{array}{l}\text { Anfotericina B liposómico } \\
\text { Anfotericina B deoxicolato } \\
\text { Fluconazol } \\
\text { Voriconazol }\end{array}$ \\
\hline $\begin{array}{l}\text { Candidemia por } \mathrm{C} \text {. } \\
\text { parapsilosis }\end{array}$ & Fluconazol & $\begin{array}{l}\text { Voriconazol } \\
\text { Anfotericina B liposómico } \\
\text { Anfotericina B deoxicolato }\end{array}$ \\
\hline $\begin{array}{l}\text { Candidemia con } \\
\text { compromiso ocular }\end{array}$ & Fluconazol & Voriconazol \\
\hline $\begin{array}{l}\text { Candidemia con } \\
\text { compromiso del sistema } \\
\text { nervioso central }\end{array}$ & Voriconazol & Anfotericina B deoxicolato \\
\hline
\end{tabular}

Cuadro 5. Dosis recomendadas y efectos adversos de medicamentos utilizados en el manejo de la candidemia

\begin{tabular}{|c|c|c|c|}
\hline Medicamento & Dosis & Efectos adversos frecuentes & Observaciones \\
\hline $\begin{array}{l}\text { Deoxicolato de } \\
\text { anfotericina B }\end{array}$ & 0,7-1 mg/kg/día & $\begin{array}{l}\text { Falla renal, hipopotasemia, } \\
\text { fiebre y otros efectos de la } \\
\text { infusión }\end{array}$ & $\begin{array}{l}\text { La anfotericina se debe acompañar de una } \\
\text { hidratación apropiada antes de la infusión y de } \\
\text { acetaminofén o meperidina como medicación } \\
\text { previa para prevenir reacciones a la infusión. } \\
\text { La infusión es prolongada y se puede hacer } \\
\text { de forma continua. Requiere monitorización } \\
\text { de la función renal y del nivel de potasio. }\end{array}$ \\
\hline $\begin{array}{l}\text { Anfotericina B } \\
\text { liposómica }\end{array}$ & 3-5 mg/kg/día & $\begin{array}{l}\text { Falla renal con menor } \\
\text { frecuencia, disnea y otros } \\
\text { efectos de la infusión }\end{array}$ & $\begin{array}{l}\text { La infusión inicial es de } 2 \mathrm{~h} \text {, y puede acortarse } \\
\text { en pacientes que lo toleren. Requiere } \\
\text { monitorización de la función renal y los niveles } \\
\text { de potasio. }\end{array}$ \\
\hline $\begin{array}{l}\text { Anfotericina B } \\
\text { complejo lipídico }\end{array}$ & $5 \mathrm{mg} / \mathrm{kg} / \mathrm{día}$ & $\begin{array}{l}\text { Falla renal menos frecuente, } \\
\text { disnea y otros efectos de la } \\
\text { infusión }\end{array}$ & $\begin{array}{l}\text { Su uso está limitado a pacientes intolerantes } \\
\text { al deoxicolato de anfotericina B }\end{array}$ \\
\hline Anidulafungina & $\begin{array}{l}100 \mathrm{mg} / \text { día, dosis inicial de } \\
200 \mathrm{mg}\end{array}$ & Limitados & $\begin{array}{l}\text { Menor interacción medicamentosa } \\
\text { con rifampicina, carbamazepina e } \\
\text { inmunosupresores }\end{array}$ \\
\hline Caspofungina & $\begin{array}{l}50 \text { mg/día, dosis inicial de } 70 \\
\text { mg }\end{array}$ & Limitados & $\begin{array}{l}\text { Interacciones medicamentosas diversas } \\
\text { (ciclosporina, carbamazepina, rifampicina y } \\
\text { otros); no usar en falla hepática grave }\end{array}$ \\
\hline Micafungina & 100 mg/día & $\begin{array}{l}\text { Dolor abdominal, náuseas, } \\
\text { diarrea }\end{array}$ & \\
\hline Fluconazol & $\begin{array}{l}400 \mathrm{mg} / \text { día, dosis inicial de } \\
800 \mathrm{mg}\end{array}$ & Elevación de transaminasas & Requiere ajuste a la función renal \\
\hline Voriconazol & $\begin{array}{l}4 \mathrm{mg} / \mathrm{kg} \text { cada } 12 \mathrm{~h} \text {, dosis } \\
\text { inicial de } 6 \mathrm{mg} / \mathrm{kg} \text { en } 2 \text { dosis } \\
\text { para personas de }>40 \mathrm{~kg}\end{array}$ & $\begin{array}{l}\text { Elevación de transaminasas, } \\
\text { cambios en la visión de colores }\end{array}$ & $\begin{array}{l}\text { Niveles variables, especialmente con } \\
\text { administración oral }\end{array}$ \\
\hline
\end{tabular}

Reproducido de: Cortés JA, Prada G. Protocolo de estudio y manejo de pacientes con candidiasis sistémica en adultos. Infectio. 2012;16(Supl 3):118-22. 
diferencias importantes en la farmacocinética (40). Se destaca que la anidulafungina tiene un menor riesgo de interacciones farmacológicas. El tratamiento se debe adecuar según los patrones de sensibilidad obtenidos con los cultivos (38).

Las guías europeas de manejo (29) recomiendan las equinocandinas como primera línea de tratamiento de la candidemia en pacientes sin neutropenia y plantean que hay menos datos en favor del fluconazol. Estas guías destacan el bajo riesgo de interacciones de la anidulafungina comparada con la caspofungina. Se ha observado que las equinocandinas tienen efecto sobre las especies de Candida en biopelículas (41), lo cual podría tener un impacto en el tratamiento de las infecciones relacionadas con dispositivos o con la endocarditis. También, se han observado diferencias entre las equinocandinas en las biopelículas según la especie involucrada (42).

En general, en todas las guías (cuadro 4) se proponen las equinocandinas como tratamiento de primera línea de la candidemia y se considera la anidulafungina una opción válida para el manejo pacientes neutropénicos y sin neutropenia (2), tal como se ha recomendado también en Brasil (43).

En un análisis de costo-efectividad en el Reino Unido basado en un metaanálisis sobre la candidemia, en el cual se comparó la anidulafungina con la caspofungina, la micafungina y el fluconazol, se observó una mayor tasa de mejoría con todas las equinocandinas comparadas con el fluconazol. La tasa de supervivencia con anidulafungina fue mayor comparada con la de todos los demás medicamentos (44). En otro metaanálisis, también se demostró la superioridad de las equinocandinas (45).

La interpretación de los estudios científicos sobre el tema es compleja debido a que el número de estudios clínicos disponibles es pequeño y no incluyen todas las posibles comparaciones directas (46-48). Se estima que la anfotericina y las equinocandinas tienen una eficacia similar, con un mejor perfil de seguridad de las equinocandinas. El problema más importante de la anfotericina, aunque no el único, es la gran frecuencia de falla renal aguda asociada con el tratamiento. Las formas lipídicas de la anfotericina, especialmente la liposómica, han disminuido la frecuencia de este problema (49). De todas maneras, se recomienda abstenerse de usar las anfotericinas en pacientes con alto riesgo de falla renal o falla renal establecida (50).

Dicho esto, es importante recordar las situaciones en las que los azoles son mejores que las equinocandinas, incluidos los casos con compromiso ocular o del sistema nervioso central (cuadro 4). Hay controversia sobre el tratamiento de las infecciones por $C$. parapsilopsis con las equinocandinas, debido a que su concentración inhibitoria mínima es más elevada en comparación con la encontrada con otras especies de Candida. Sin embargo, en los estudios clínicos no se ha determinado una diferencia estadísticamente significativa a favor de ninguno de los productos (51). Tampoco es claro si hay diferencias en la eficacia de las diversas equinocandinas debidas a su farmacocinética (40); el beneficio potencial podría darse en la selección de cepas resistentes y las interacciones medicamentosas. La frecuencia de descontinuación fue más elevada con la caspofungina, aunque mucho menor comparada con la anfotericina $\mathrm{B}(3,8 \%$ Vs. más de $10 \%)(52)$. 


\section{Resistencia a los antifúngicos}

Se han observado diferentes tasas de resistencia a los antimicóticos en el país. Las variaciones en la resistencia al fluconazol se deben fundamentalmente al tipo de técnica utilizada y a los pacientes observados. Las tasas de resistencia también varían según la especie identificada, ya que $C$. krusei es intrínsecamente resistente al fluconazol y $C$. glabrata tiene sensibilidad disminuida frente a este $(1,53)$. En el estudio latinoamericano citado, se utilizaron técnicas de referencia (dilución en caldo) y es la muestra de aislamientos más grande de la región. En este estudio, la resistencia al fluconazol y a las equinocandinas fue muy baja (2). Sin embargo, se han identificado aislamientos con mutaciones de resistencia en nuestro medio (54).

\section{Conclusiones}

En Colombia, la candidemia es una entidad relativamente frecuente, con tasas de incidencia y prevalencia más altas que en otras partes del mundo; es la quinta causa de infección del torrente sanguíneo en las unidades de cuidados intensivos y representa el $88 \%$ de las infecciones fúngicas en pacientes hospitalizados, con una mortalidad que oscila entre el 36 y el $40 \%$.

El diagnóstico de la candidiasis debe basarse en la identificación de grupos de riesgo con mayor frecuencia de la infección. Los hemocultivos permiten la identificación de la especie y la posibilidad de establecer la sensibilidad antifúngica, sin embargo, están limitados por su poca sensibilidad. Las nuevas estrategias incluyen técnicas moleculares que deben implementarse ampliamente para mejorar el diagnóstico, disminuir la mortalidad y reducir el uso incorrecto de los antifúngicos.

El principal factor de mejoría del pronóstico es el inicio temprano del tratamiento antifúngico (33). Las guías desarrolladas con base en revisiones sistemáticas de la literatura recomiendan el uso de las equinoandinas en los pacientes inestables. Otras alternativas incluyen el fluconazol y la anfotericina $B$, aunque con limitaciones en la eficacia y la seguridad, respectivamente.

\section{Referencias}

1. Cortés JA, Reyes P, Gómez CH, Cuervo SI, Rivas P, Casas CA, et al. Clinical and epidemiological characteristics and risk factors for mortality in patients with candidemia in hospitals from Bogotá, Colombia. Braz J Infect Dis. 2014;18:631-7. https://doi.org/10.1016/j.bjid.2014.06.009

2. Nucci M, Thompson-Moya L, Guzmán-Blanco M, Tiraboschi IN, Cortés JA, Echevarría J, et al. Recommendations for the management of candidemia in adults in Latin America. Latin America Invasive Mycosis Network. Rev Iberoam Micol. 2013;30:179-88. https://doi.org/10.1016/j.riam.2013.05.007

3. Zuluaga-Rodríguez A, De Bedout-Gómez C, Agudelo-Restrepo C, Hurtado-Parra H, ArangoArteaga M, Restrepo-Moreno A, et al. Sensibilidad a fluconazol y voriconazol de especies de Candida aisladas de pacientes provenientes de unidades de cuidados intensivos en Medellín, Colombia (2001-2007). Rev Iberoam Micol. 2010;27:125-9. https://doi.org/10.1016/j.riam.2010.04.001

4. Cortés JA, Reyes P, Gómez C, Buitrago G, Leal AL y Group G. Fungal bloodstream infections in tertiary care hospitals in Colombia. Rev Iberoam Micol. 2011;28:74-8. https://doi.org/10.1016/j.riam.2010.12.002

5. Nucci M, Queiroz-Telles F, Alvarado-Matute T, Tiraboschi IN, Cortés J, Zurita J, et al. Epidemiology of candidemia in Latin America: A laboratory-based survey. PLoS ONE. 2013;8:e59373. https://doi.org/10.1371/journal.pone.0059373

6. Berrío I, Maldonado N, De Bedout C, Arango K, Cano LE, Valencia Y, et al. Comparative study of Candida spp. isolates: Identification and echinocandin susceptibility in isolates obtained from blood cultures in 15 hospitals in Medellín, Colombia. J Glob Antimicrob Resist. 2018;13:254-60. https://doi.org/10.1016/j.jgar.2017.11.010 
7. Paramythiotou E, Frantzeskaki F, Flevari A, Armaganidis A, Dimopoulos G. Invasive fungal infections in the ICU: How to approach, how to treat. Molecules. 2014;19:1085-119. https://doi.org/10.3390/molecules19011085

8. Scudeller L, Viscoli C, Menichetti F, del Bono V, Cristini F, Tascini C, et al. An Italian consensus for invasive candidiasis management (ITALIC). Infection. 2014;42:263-79. https://doi.org/10.1007/s15010-013-0558-0

9. Eggimann P, Garbino J, Pittet D. Management of Candida species infections in critically ill patients. Lancet Infect Dis. 2003;3:772-85. https://doi.org/10.1016/S1473-3099(03)00831-4

10. Cortés JA, Corrales IF. Invasive candidiasis: Epidemiology and risk factors in fungal infection. Londres: Intech Open; 2019:33-46. https://doi.org/10.5772/intechopen.81813

11. Ortiz-Ruiz G, Osorio J, Valderrama S, Álvarez D, Elias-Díaz R, Calderón J, et al. Risk factors for candidemia in non-neutropenic critical patients in Colombia. Med Intensiva. 2016;40:139-44. https://doi.org/10.1016/j.medin.2015.08.001

12. De Bedout C, Gómez B. Candida y candidiasis invasora: un reto continuo para su diagnóstico temprano. Infectio. 2010;14:s159-71. https://doi.org/10.1016/S0123-9392(10)70133-8

13. Orozco P, Cortés J, Parra C. Colonización por levaduras en recién nacidos y personal de salud en la unidad de cuidados intensivos neonatales de un hospital universitario en Bogotá, Colombia. Rev Iberoam Micol. 2009;26:108-11 https://doi.org/10.1016/S1130-1406(09)70020-8

14. Díaz-Granados CA, Martínez A, Deaza C, Valderrama S. An outbreak of Candida spp. bloodstream infection in a tertiary care center in Bogotá, Colombia. Braz J Infect Dis. 2008;12:390-4.

15. Asmundsdottir LR, Erlendsdottir H, Haraldsson G, Guo H, Xu J, Gottfredsson M. Molecular epidemiology of candidemia: Evidence of clusters of smoldering nosocomial infections. Clin Infect Dis. 2008;47:e17-24. https://doi.org/10.1086/589298

16. Desai JV, Mitchell AP. Candida albicans biofilm development and its genetic control. Microbiol Spectr. 2015;3(3):MB-0005-2014. https://doi.org/10.1128/microbiolspec.MB-0005-2014

17. Cortés JA, Jaimes JA, Leal AL. Incidencia y prevalencia de candidemia en pacientes críticamente enfermos en Colombia. Rev Chil Infectol. 2013;30:599-604. https://doi.org/10.4067/S0716-10182013000600004

18. Lockhart SR, Etienne KA, Vallabhaneni S, Farooqi J, Chowdhary A, Govender NP, et al. Simultaneous emergence of multidrug-resistant Candida auris on 3 continents confirmed by whole-genome sequencing and epidemiological analyses. Clin Infect Dis. 2017;64:134-40. https://doi.org/10.1093/cid/ciw691

19. Parra-Giraldo CM, Valderrama SL, Cortés-Fraile G, Garzón JR, Ariza BE, Morio F, et al. First report of sporadic cases of Candida auris in Colombia. Int J Infect Dis. 2018;69:63-7. https://doi.org/10.1016/j.jijid.2018.01.034

20. Escandón P, Chow NA, Cáceres DH, Gade L, Berkow EL, Armstrong P, et al. Molecular epidemiology of Candida auris in Colombia reveals a highly related, countrywide colonization with regional patterns in amphotericin B resistance. Clin Infect Dis. 2019;68:1521. https://doi.org/10.1093/cid/ciy411

21. Morales-López SE, Parra-Giraldo CM, Ceballos-Garzón A, Martínez HP, Rodríguez GJ, Álvarez-Moreno CA, et al. Invasive infections with multidrug-resistant yeast Candida auris, Colombia. Emerg Infect Dis. 2017;23:162-4. https://doi.org/10.3201/eid2301.161497

22. Motoa G, Muñoz JS, Oñate J, Pallares CJ, Hernández C, Villegas MV. Epidemiology of Candida isolates from intensive care units in Colombia from 2010 to 2013. Rev lberoam Micol. 2017;34:17-22. https://doi.org/10.1016/j.riam.2016.02.006

23. Thomaz DY, de Almeida JN Jr, Lima GM, Nunes MO, Camargo CH, Grenfell RC, et al. An azole-resistant Candida parapsilosis outbreak: clonal persistence in the intensive care unit of a Brazilian teaching hospital. Front Microbiol. 2018;9:2997. https://doi.org/10.3389/fmicb.2018.02997

24. Cortés JA, Prada G. Protocolo de estudio y manejo de pacientes con candidiasis sistémica en adultos. Infectio. 2012;16:118-22. https://doi.org/10.1016/S0123-9392(12)70037-1

25. Ahmadi A, Ardehali SH, Beigmohammadi MT, Hajiabdolbaghi M, Hashemian SM, Kouchek $\mathrm{M}$, et al. Invasive candidiasis in intensive care unit; consensus statement from an Iranian panel of experts, July 2013. JRSM Open. 2014;5:2042533313517689. https://doi.org/10.1177/2042533313517689 
26. Pappas PG, Kauffman CA, Andes D, Benjamin DK Jr, Calandra TF, Edwards JE Jr, et al. Clinical practice guidelines for the management of candidiasis: 2009 update by the Infectious Diseases Society of America. Clin Infect Dis. 2009;48:503-35. https://doi.org/10.1086/596757

27. Díaz J, Urrego J, Moreno J, Huérfano C, Prieto V. Análisis de costo-efectividad de anidulafungina en el tratamiento de la candidiasis invasiva en Colombia. Value Health. 2015;18:A870. https://doi.org/10.1016/j.jval.2015.09.010

28. Kami M, Machida U, Okuzumi K, Matsumura T, Mori Si S, Hori A, et al. Effect of fluconazole prophylaxis on fungal blood cultures: An autopsy-based study involving 720 patients with haematological malignancy. $\mathrm{Br} \mathrm{J}$ Haematol. 2002;117:40-6. https://doi.org/10.1046/j.1365-2141.2002.03414.x

29. Cornely OA, Bassetti M, Calandra T, Garbino J, Kullberg BJ, Lortholary O, et al. ESCMID* guideline for the diagnosis and management of Candida diseases 2012: Non-neutropenic adult patients. Clin Microbiol Infect. 2012;18(Suppl.7):19-37. https://doi.org/10.1111/1469-0691.12039

30. León C, Ruiz-Santana S, Saavedra P, Galván B, Blanco A, Castro C, et al. Usefulness of the "Candida score" for discriminating between Candida colonization and invasive candidiasis in non-neutropenic critically ill patients: A prospective multicenter study. Crit Care Med. 2009;37:1624-33. https://doi.org/10.1097/CCM.0b013e31819daa14

31. Mylonakis E, Clancy CJ, Ostrosky-Zeichner L, Garey KW, Alangaden GJ, Vázquez JA, et al. T2 magnetic resonance assay for the rapid diagnosis of candidemia in whole blood: A clinical trial. Clin Infect Dis. 2015;60:892-9. https://doi.org/10.1093/cid/ciu959

32. Berdal JE, Haagensen R, Ranheim T, Bjornholt JV. Nosocomial candidemia; risk factors and prognosis revisited; 11 years experience from a Norwegian secondary hospital. PLoS ONE. 2014;9:e103916. https://doi.org/10.1371/journal.pone.0103916

33. Garey KW, Rege M, Pai MP, Mingo DE, Suda KJ, Turpin RS, et al. Time to initiation of fluconazole therapy impacts mortality in patients with candidemia: A multi-institutional study. Clin Infect Dis. 2006;43:25-31. https://doi.org/10.1086/504810

34. Aguado JM, Ruiz-Camps I, Muñoz P, Mensa J, Almirante B, Vázquez L, et al. Recomendaciones sobre el tratamiento de la candidiasis invasiva y otras infecciones por levaduras de la Sociedad Española de Enfermedades Infecciosas y Microbiología Clínica (SEIMC). Actualización 2011. Enferm Infecc Microbiol Clin. 2011;29:345-61. https://doi.org/10.1016/j.eimc.2011.01.008

35. Kullberg BJ, Verweij PE, Akova M, Arendrup MC, Bille J, Calandra T, et al. European expert opinion on the management of invasive candidiasis in adults. Clin Microbiol Infect. 2011;17(Suppl.5):1-12. https://doi.org/10.1111/j.1469-0691.2011.03615.x

36. Rodríguez-Adrián LJ, King RT, Tamayo-Derat LG, Miller JW, García CA, Rex JH. Retinal lesions as clues to disseminated bacterial and candidal infections: Frequency, natural history, and etiology. Medicine (Baltimore). 2003;82:187-202. https://doi.org/10.1097/01.md.0000076008.64510.f1

37. Centers for Disease Control and Prevention. Invasive candidiasis. Fecha de consulta: 31 marzo de 2018. Disponible en: https://www.cdc.gov/fungal/diseases/candidiasis/invasive/ index.html

38. Pappas PG, Kauffman CA, Andes DR, Clancy CJ, Marr KA, Ostrosky-Zeichner L, et al. Clinical Practice Guideline for the Management of Candidiasis: 2016 Update by the Infectious Diseases Society of America. Clin Infect Dis. 2016;62:e1-50. https://doi.org/10.1093/cid/civ933

39. Oñate J, Rivas J, Pallares C, Saavedra C, Martínez E, Coronell W, et al. Colombian consensus on the diagnosis, treatment, and prevention of Candida spp. disease in children and adults. Infectio. 2019;23:271-304.

40. Cortés JA, Russi JA. Equinocandinas. Rev Chil Infectol. 2011;28:529-36. https://doi.org/10.4067/S0716-10182011000700004

41. Marcos-Zambrano LJ, Gómez-Perosanz M, Escribano P, Zaragoza O, Bouza E, Guinea J. Biofilm production and antibiofilm activity of echinocandins and liposomal amphotericin $B$ in echinocandin-resistant yeast species. Antimicrob Agents Chemother. 2016;60:3579-86. https://doi.org/10.1128/AAC.03065-15 
42. Marcos-Zambrano LJ, Escribano P, Bouza E, Guinea J. Comparison of the antifungal activity of micafungin and amphotericin B against Candida tropicalis biofilms. J Antimicrob Chemother. 2016;71:2498-501. https://doi.org/10.1093/jac/dkw162

43. Colombo AL, Guimaraes T, Camargo LF, Richtmann R, Queiroz-Telles F, Salles MJ, et al. Brazilian guidelines for the management of candidiasis - a joint meeting report of three medical societies: Sociedade Brasileira de Infectologia, Sociedade Paulista de Infectologia and Sociedade Brasileira de Medicina Tropical. Braz J Infect Dis. 2013;17:283-312. https://doi.org/10.1016/j.bjid.2013.02.001

44. Auzinger G, Playford EG, Graham CN, Knox HN, Weinstein D, Kantecki M, et al. Costeffectiveness analysis of anidulafungin for the treatment of candidaemia and other forms of invasive candidiasis. BMC Infect Dis. 2015;15:463. https://doi.org/10.1186/s12879-015-1143-1

45. Andes DR, Safdar N, Baddley JW, Playford G, Reboli AC, Rex JH, et al. Impact of treatment strategy on outcomes in patients with candidemia and other forms of invasive candidiasis: A patient-level quantitative review of randomized trials. Clin Infect Dis. 2012;54:1110-22. https://doi.org/10.1093/cid/cis021

46. Pappas PG, Rotstein CM, Betts RF, Nucci M, Talwar D, De Waele JJ, et al. Micafungin versus caspofungin for treatment of candidemia and other forms of invasive candidiasis. Clin Infect Dis. 2007;45:883-93. https://doi.org/10.1086/520980

47. Kubiak DW, Bryar JM, McDonnell AM, Delgado-Flores JO, Mui E, Baden LR, et al. Evaluation of caspofungin or micafungin as empiric antifungal therapy in adult patients with persistent febrile neutropenia: A retrospective, observational, sequential cohort analysis. Clin Ther. 2010;32:637-48. https://doi.org/10.1016/j.clinthera.2010.04.005

48. Yan T, Li SL, Wang DX. Comparison between micafungin and caspofungin for the empirical treatment of severe intra-abdominal infections in surgical intensive care patients. Zhonghua Yi Xue Za Zhi. 2016;96:2301-6. https://doi.org/10.3760/cma.j.issn.0376-2491.2016.29.004

49. Botero MC, Puentes-Herrera M, Cortés JA. Formas lipídicas de anfotericina. Rev Chil Infectol. 2014;31:518-27. https://doi.org/10.4067/S0716-10182014000500002

50. Cortés JA, Soto R, Ãlvarez CA, Buitrago G, Camargo RD, Cataño JC, et al. Consenso de uso de antimicrobianos en pacientes críticamente enfermos con falla renal o en riesgo de padecerla. Infectio. 2011;15:49-63. https://doi.org/10.1016/S0123-9392(11)70076-5

51. Reboli AC, Rotstein C, Pappas PG, Chapman SW, Kett DH, Kumar D, et al. Anidulafungin versus fluconazole for invasive candidiasis. N Engl J Med. 2007;356:2472-82. https://doi.org/10.1056/NEJMoa066906

52. Wang JL, Chang CH, Young-Xu Y, Chan KA. Systematic review and meta-analysis of the tolerability and hepatotoxicity of antifungals in empirical and definitive therapy for invasive fungal infection. Antimicrob Agents Chemother. 2010;54:2409-19. https://doi.org/10.1128/AAC.01657-09

53. Maldonado NA, Cano LE, De Bedout C, Arbeláez CA, Roncancio G, Tabares AM, et al. Association of clinical and demographic factors in invasive candidiasis caused by fluconazoleresistant Candida species: A study in 15 hospitals, Medellín, Colombia 2010-2011. Diagn Microbiol Infect Dis. 2014;79:280-6. https://doi.org/10.1016/j.diagmicrobio.2014.02.003

54. Rodríguez-Leguizamón G, Fiori A, Lagrou K, Gaona MA, Ibañéz M, Patarroyo MA, et al. New echinocandin susceptibility patterns for nosocomial Candida albicans in Bogota, Colombia, in ten tertiary care centres: An observational study. BMC Infect Dis. 2015;15:108. https://doi.org/10.1186/s12879-015-0840-0 